\title{
Analysis of the molecular subtypes of preoperative core needle biopsy and surgical specimens in invasive breast cancer
}

\author{
Ye Sul Jeong ${ }^{1}$, Jun Kang ${ }^{1}$, Jieun Lee ${ }^{2,3}$, Tae-Kyung Yoo ${ }^{4}$, Sung Hun Kim5, Ahwon Lee ${ }^{1,3}$ \\ 'Department of Hospital Pathology, ${ }^{2}$ Division of Medical Oncology, Department of Internal Medicine, Seoul St. Mary's Hospital, College of Medicine, \\ The Catholic University of Korea, Seoul; \\ ${ }^{3}$ Cancer Research Institute, The Catholic University of Korea, Seoul; \\ Departments of ${ }^{4}$ Surgery and ${ }^{5}$ Radiology, Seoul St. Mary's Hospital, College of Medicine, The Catholic University of Korea, Seoul, Korea
}

\begin{abstract}
Background: Accurate molecular classification of breast core needle biopsy (CNB) tissue is important for determining neoadjuvant systemic therapies for invasive breast cancer. The researchers aimed to evaluate the concordance rate (CR) of molecular subtypes between CNBs and surgical specimens. Methods: This study was conducted with invasive breast cancer patients who underwent surgery after CNB at Seoul St. Mary's Hospital between December 2014 and December 2017. Estrogen receptor (ER), progesterone receptor (PR), human epidermal growth factor receptor 2 (HER2), and Ki67 were analyzed using immunohistochemistry. ER and PR were evaluated by Allred score (0-8). HER2 was graded from 0 to +3 , and all $2+$ cases were reflex tested with silver in situ hybridization. The labeling index of Ki67 was counted by either manual scoring or digital image analysis. Molecular subtypes were classified using the above surrogate markers. Results: In total, 629 patients were evaluated. The CRs of ER, PR, HER2, and Ki67 were 96.5\% (kappa, 0.883; p<.001), 93.0\% (kappa, 0.824; $p<.001$ ), 99.7\% (kappa, 0.988; $p<.001$ ), and 78.7\% (kappa, 0.577; $p<.001$ ), respectively. Digital image analysis of Ki67 in CNB showed better concordance with Ki67 in surgical specimens (CR, 82.3\%; kappa, 0.639 for digital image analysis vs. CR, 76.2\%; kappa, 0.534 for manual counting). The CRs of luminal A, luminal B, HER2, and triple negative types were $89.0 \%, 70.0 \%, 82.9 \%$, and $77.2 \%$, respectively. Conclusions: CNB was reasonably accurate for determining ER, PR, HER2, Ki67, and molecular subtypes. Using digital image analysis for Ki67 in CNB produced more accurate molecular classifications.
\end{abstract}

Key Words: Breast neoplasms; Core needle biopsy; Receptors, estrogen; Receptors, progesterone; Human epidermal growth factor receptor 2; Immunohistochemistry

Received: August 1, 2019 Revised: September 25, 2019 Accepted: October 14, 2019

Corresponding Author: Ahwon Lee, MD, PhD, Department of Hospital Pathology, Seoul St. Mary's Hospital, College of Medicine, The Catholic University of Korea, 222 Banpo-daero, Seocho-gu, Seoul 06591, Korea

Tel: +82-2-2258-1621, Fax: +82-2-2258-1627, E-mail: klee@catholic.ac.kr

Breast cancer is a heterogeneous disease that is classified into four molecular subtypes: luminal A, luminal B, human epidermal growth factor receptor 2 (HER2) positive, and triple negative. Proper disease subtyping is important for predicting response to systemic therapy and prognosis. Immunohistochemistry for estrogen receptor (ER), progesterone receptor (PR), HER2, and Ki67 can be used to approximate molecular subtype classifications [1-3].

Endocrine therapy, cytotoxic therapy, and anti-HER2 therapy are used in the adjuvant setting for breast cancer, and molecular subtyping is used to decide which therapies are appropriate [3]. Neoadjuvant therapy is important in advanced breast cancer as it can reduce tumor size, facilitating breast conserving surgery, and decrease distant micrometastasis. Neoadjuvant therapy may also facilitate axillary preservation [4-7]. After receiving neoadjuvant therapy, some patients achieve pathologic complete response (pCR), at which point, core needle biopsy (CNB) tissue is the only specimen for obtaining information about prognostic markers that could impact therapeutic plans. Although some patients still have tumor burden after neoadjuvant therapy, CNB might be the only specimen available in which to evaluate histological markers because neoadjuvant therapy can alter histological grade, expression of hormonal receptors, HER2 status, and Ki67. Consequently, assessing the concordance rate (CR) of marker status between $\mathrm{CNB}$ and surgical specimen is crucial [8,9]. It has been reported that $\mathrm{CRs}$ of biomarker status between $\mathrm{CNB}$ and surgi- 
cal specimen were high but variable: 79\%-100\% [10]. However, differences in $\mathrm{Ki} 67$ status according to manual or digital image analysis and the impact on subsequent molecular subtyping have not been adequately investigated. The aim of this study was to evaluate and analyze CRs of hormone receptors, HER2, Ki67, and molecular subtypes in invasive breast cancer patients between CNB and surgical specimens.

\section{MATERIALS AND METHODS}

\section{Patient population and tissue samples}

This study retroactively analyzed clinical data from cancer patients who underwent surgery after diagnosis by CNB at Seoul St. Mary's Hospital. Clinicopathologic data of age; histologic type; grade; operation method; pTNM stage; immunohistochemical staining results of ER, PR, HER2, and $\mathrm{Ki} 67$; and whether digital image analysis was used to analyze results of Ki67 staining and silver in situ hybridization (SISH) for HER2 were obtained through medical records and pathologic reports. CNBs were mostly performed by ultrasound guidance with 14-gauge needles; 4-5 pieces were received for mass forming lesions and $>7$ pieces for microcalcifications. Especially when microcalcification was noted, an ultrasound-guided or stereotactic mammotome biopsy with 11-gauge needles was performed, and at least 12 pieces of core tissue were obtained for pathologic examination. All specimens were routinely processed and diagnosed according to national and international guidelines. Briefly, CNB specimens were fixed in 10\% neutral formalin for 8-11 hours, embedded in paraffin, and sectioned at 4- $\mu \mathrm{m}$ thickness; hematoxylin and eosin (H\&E) staining was subsequently performed. Surgical specimens were cut into $0.5-1-\mathrm{cm}$-thick sections after the operation and fixed in $10 \%$ neutral formalin for 8-24 hours; H\&E staining was subsequently performed. Patients who were diagnosed with ductal carcinoma in situ, microinvasive breast cancer, or had received preoperative systemic therapy were excluded.

\section{Evaluation of ER, PR, HER2, and Ki67}

Immunohistochemistry (IHC) for ER, PR, HER2, and Ki67 was performed following the instructions of the pathology laboratory manual. Briefly, ER, PR, HER2, and Ki67 IHC staining was performed on an automated Ventana BenchmarkXT slide stainer (Ventana, Tucson, AZ, USA), using primary antibodies against ER (prediluted, SP1, Ventana), PR (prediluted, 1E2, Ventana), HER2 (prediluted, 4B5, Ventana), and Ki67 (prediluted, MIB-1, Ventana). The HER2 SISH assay was performed with INFORM HER2 DNA probes (Ventana) on the Ventana
BenchMarkXT automated slide stainer according to the manufacturer's protocols.

The Allred scoring system was used to interpret ER and PR staining [11]. The proportion of positive-stained tumor cells (the proportion score) was rated as follows: 0 , no cells stained positive; $1,0 \%-1 \%$ positive; $2,1 \%-10 \%$ positive; $3,10 \%-33 \%$ positive; 4, 33\%-66\% positive; and 5, 66\%-100\% positive. Intensity was scored on the basis of average staining intensity: 0 , negative; 1 , weak; 2 , intermediate; and 3, strong. The sum of the proportion and intensity scores is referred to as the Allred score, and scores $>2$ are defined as positive [11]. HER2 status was scored from $0-3+$ by IHC, where 0 was defined as no staining or membrane staining that was incomplete, faint, or barely perceptible in $\leq 10 \%$ of invasive tumor cells; $1+$ was defined as $>10 \%$ invasive tumor cells with incomplete membrane staining that was faint or barely perceptible; $2+$ was defined as $>10 \%$ invasive tumor cells with complete weak to moderate membrane staining (considered equivocal); and $3+$ was defined as $>10 \%$ invasive tumor cells with complete, intense membrane staining (positive according to American Society of Clinical Oncology [ASCO]/College of American Pathologists [CAP] guidelines) $[12,13]$. Cases of scores 0 and $1+$ were considered negative, while those $2+$ were further evaluated by reflex HER2 SISH to confirm HER2 gene amplification.

Ki67 was evaluated according to the percentage of positivelystained invasive tumor cells of any intensity by pathologists manually or using an automated digital image analysis system. The "eyeballed" estimation method, which is approximate counting throughout the immunostained slide, was used for manual counting [14]. For Ki67 digital image analysis, slides were scanned by an iScan Coreo slide scanner with a $20 \times$ objective (Ventana), and invasive tumor components were analyzed using Virtuoso software (Ventana). At least three high-power fields (400x) including hot spots where the highest Ki67 staining area and two average intensity areas of Ki67 staining were selected. More than 1,000 tumor cells were counted according to the International Ki67 in Breast Cancer Working Group [15]. In the case of $\mathrm{CNB}$ with a heterogeneous $\mathrm{Ki} 67$ staining pattern, researchers attempted to select all tumor cells to evaluate $\mathrm{Ki} 67$ expression. Cases in which less than 500 tumor cells were present in CNB were excluded from digital image analysis. Digital image analysis of Ki67 was performed in 41\% (260/629) of CNBs and all but 14 surgical specimens. The cutoff value for Ki67 was $20 \%$.

\section{Molecular subtype classifications}

Molecular subtypes were classified as follows: luminal A: ER- 
and/or PR-positive, HER2-negative, Ki67 $\leq 20 \%$; luminal B: ER- and/or PR-positive, HER2-negative, Ki67 > 20\% or ERand/or PR-positive, HER2-positive, any Ki67; HER2-positive: ER- and PR-negative, HER2-positive; and triple-negative (basallike): ER-, PR-, and HER2-negative. Subsequently, luminal B was further divided as luminal B-HER2-negative (ER- and/or PR-positive, HER2-negative, Ki67 > 20\%) and luminal BHER2-positive (ER- and/or PR-positive, HER2-positive, any $\operatorname{Ki67)}[2,3]$.

\section{Statistical analysis}

CRs of receptor status and molecular subtypes between CNB and surgical specimens were calculated as percentage and Kappa value. K-values $<0.20$ were correlated with poor agreement, 0.21-0.40 fair agreement, $0.41-0.60$ moderate agreement, $0.61-0.80$ good agreement, and $0.81-1.00$ very good agreement. p-values were calculated using the chi-square test or Fisher exact test, and p-values of $<0.05$ were considered significant. Statistical analyses were performed using IBM SPSS Statistics software ver. 24.0 for Windows (IBM Corp., Armonk, NY, USA). Sankey diagrams depicting changes in Allred scores were computed in SankeyMATIC (http://sankeymatic.com).

\section{Ethics statement}

The protocol of the study was approved by the Institutional Review Board of The Catholic Medical Center, which waived the requirement for informed consent (approval KC19RESI0333).

\section{RESULTS}

\section{Patient characteristics}

In total, 629 patients were included in the study. The median age was 53 (range, 23 to 89 years). All patients received either breast conserving surgery $(70.4 \%)$ or mastectomy $(29.6 \%)$. The most common pathological tumor type was invasive carcinoma of no special type $(80.1 \%)$ (Tables 1,2$)$.

\section{Concordance of ER, PR, HER2, and Ki67}

The CRs of surgical specimens with CNBs for ER and PR were $96.5 \%$ (kappa, 0.883; p <.001) and 93.0\% (kappa, 0.824; $\mathrm{p}<.001$ ), respectively (Table 3 ). The concordance of PR was slightly lower than that of ER. Changes in Allred score for ER and PR are shown in Figs. 1 and 2. The CR of surgical specimens with $\mathrm{CNBs}$ for hormone receptor status was $95.1 \%$, with very good agreement (kappa, 0.824; $\mathrm{p}<.001$ ). The CR of HER2
Table 1. Patient and tumor characteristics

\begin{tabular}{lc}
\hline Variable & No. (\%) \\
\hline Age (yr), median (range) & $53(23-89)$ \\
$\leq 50$ & $279(44.4)$ \\
$>50$ & $350(55.6)$ \\
Surgery type & \\
Breast consenving surgery & $443(70.4)$ \\
Mastectomy & $186(29.6)$ \\
Pathological type & \\
Invasive carcinoma of no special type & $504(80.1)$ \\
Invasive lobular carcinoma & $45(7.2)$ \\
Mucinous carcinoma & $24(3.8)$ \\
Carcinoma with medullary feature & $13(2.1)$ \\
Metaplastic carcinoma & $8(1.3)$ \\
Minor pathological type & $35(5.6)$ \\
Histologic grade (Nottingham histologic grading) & \\
Grade 1 & $143(22.7)$ \\
Grade 2 & $286(45.5)$ \\
Grade 3 & $200(31.8)$ \\
Pathologic T category & \\
pT1 & $374(59.5)$ \\
pT2 & $242(38.5)$ \\
pT3 & $11(1.7)$ \\
pT4 & $2(0.3)$ \\
Pathologic N category & \\
pNX & $7(1.1)$ \\
pN0 & $429(68.2)$ \\
pN1 & $140(22.3)$ \\
pN2 & $28(4.5)$ \\
pN3 & $25(4.0)$ \\
\hline
\end{tabular}

Table 2. Minor pathological type from Table 1

\begin{tabular}{lc}
\hline Pathological type & No. (\%) \\
\hline Mixed invasive ductal and mucinous carcinoma & $7(1.1)$ \\
Invasive micropapillary carcinoma & $6(1.0)$ \\
Mixed invasive ductal and micropapillary carcinoma & $6(1.0)$ \\
Carcinoma with apocrine differentiation & $4(0.6)$ \\
Tubular carcinoma & $4(0.6)$ \\
Mixed invasive ductal and lobular carcinoma & $3(0.5)$ \\
Invasive cribriform carcinoma & $2(0.3)$ \\
Medullary carcinoma & $1(0.2)$ \\
Invasive papillary carcinoma & $1(0.2)$ \\
Mixed invasive ductal and apocrine carcinoma & $1(0.2)$ \\
\hline
\end{tabular}

IHC was $81.4 \%$, with moderate agreement (kappa, 0.591; p < .001). After reflex HER2 SISH, the CR of HER2 status was 99.7\% (kappa, 0.988; p <.001). The CR for Ki67 was $78.7 \%$ (kappa, 0.577; $\mathrm{p}<.001$, moderate agreement), which was lower than for hormone receptors and HER2 due to higher Ki67 expression in surgical specimens (Table 3).

We next analyzed whether the Ki67 counting method (automated digital image analysis system or manual scoring) affected the CR for Ki67. Among the 629 cases, 260 CNBs were analyzed 
Table 3. Concordance between CNB and surgical specimen for ER, PR, HER2, and Ki67 results

\begin{tabular}{|c|c|c|c|c|c|c|c|}
\hline \multirow{2}{*}{ Surgical specimen } & \multicolumn{3}{|c|}{ CNB } & \multirow{2}{*}{ Total (\%) } & \multirow{2}{*}{ Concordance rate } & \multirow{2}{*}{ Kappa $^{a}$} & \multirow{2}{*}{$p$-value } \\
\hline & Neg & Pos & Equi & & & & \\
\hline \multicolumn{8}{|l|}{ Hormone receptor } \\
\hline Neg & 90 & 24 & & $114(18.1)$ & & & \\
\hline Pos & 7 & 508 & & $515(81.9)$ & 95.1 & 0.824 & $<.001$ \\
\hline Total & $97(15.4)$ & $532(84.6)$ & & 629 & & & \\
\hline \multicolumn{8}{|l|}{ ER } \\
\hline Neg & 104 & 16 & & $120(19.1)$ & & & \\
\hline Pos & 6 & 503 & & $509(80.9)$ & 96.5 & 0.883 & $<.001$ \\
\hline Total & $110(17.5)$ & $519(82.5)$ & & 629 & & & \\
\hline \multicolumn{8}{|l|}{ PR } \\
\hline Neg & 150 & 28 & & 178 (28.3) & & & \\
\hline Pos & 16 & 435 & & $451(71.7)$ & 93.0 & 0.824 & $<.001$ \\
\hline Total & $166(26.4)$ & 463 (73.6) & & 629 & & & \\
\hline \multicolumn{8}{|l|}{ HER2 (IHC) } \\
\hline Neg & 396 & 1 & 69 & $466(74.1)$ & & & \\
\hline Pos & 0 & 73 & 6 & 79 (12.6) & & & \\
\hline Equi & 31 & 10 & 43 & $84(13.4)$ & 81.4 & 0.591 & $<.001$ \\
\hline Total & 427 (67.9) & $84(13.4)$ & $118(18.8)$ & 629 & & & \\
\hline \multicolumn{8}{|l|}{ HER2 (IHC+SISH) } \\
\hline Neg & 532 & 1 & & $533(84.7)$ & & & \\
\hline Pos & 1 & 95 & & 96 (15.3) & 99.7 & 0.988 & $<.001$ \\
\hline Total & $533(84.7)$ & $96(15.3)$ & & 629 & & & \\
\hline Ki67 & $\leq 20 \%$ & $>20 \%$ & & & & & \\
\hline$\leq 20 \%$ & 225 & 33 & & $258(41.0)$ & & & \\
\hline$>20 \%$ & 101 & 270 & & $371(59.0)$ & 78.7 & 0.577 & $<.001$ \\
\hline Total & $326(51.8)$ & $303(48.2)$ & & 629 & & & \\
\hline
\end{tabular}

Values are presented as number (\%) unless otherwise indicated.

CNB, core needle biopsy; ER, estrogen receptor; PR, progesterone receptor; HER2, human epidermal growth factor receptor 2; Neg, negative; Pos, positive; Equi, Equivocal; IHC, immunohistochemistry; SISH, siver in situ hybridization.

aKappa: <0.20, poor agreement; $0.21-0.40$, fair agreement; $0.41-0.60$, moderate agreement; $0.61-0.80$, good agreement; $0.81-1.00$, very good agreement.

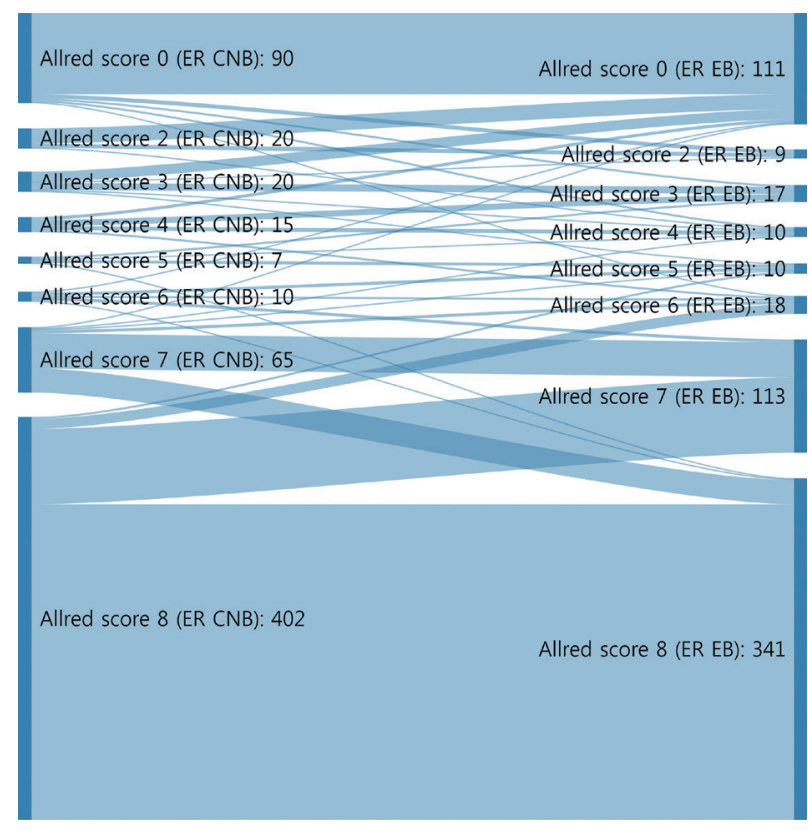

Fig. 1. Sankey diagrams depicting changes in Allred scores for estrogen receptor (ER) from core needle biopsy (CNB) to surgical specimen. EB, excisional biopsy (surgical specimen).
Allred score 0 (PR CNB): $142 \quad$ Allred score 0 (PR EB): 166

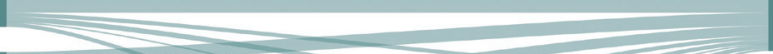

Allred score 2(PR CNB): $24 \quad$ Allred score 2 (PR EB): 12 Allred score 3 (PR CNB): 25 Allred score 4 (PR CNB): 25
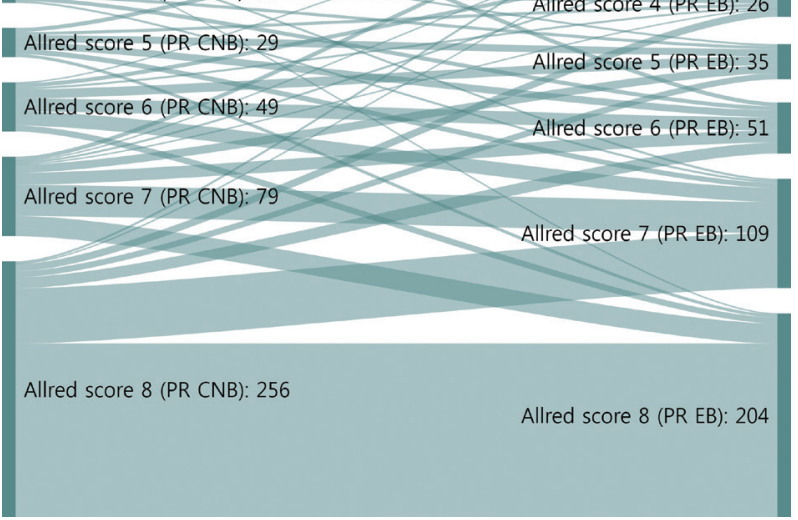

Fig. 2. Sankey diagrams depicting changes in Allred scores for progesterone receptor (PR) from core needle biopsy (CNB) to surgical specimen. EB, excisional biopsy (surgical specimen). 
with a digital image system and 369 with manual counting. When CNB Ki67s were obtained using the digital image analysis system, the $\mathrm{CR}$ for $\mathrm{Ki} 67$ was $82.3 \%$ (kappa, 0.639; p < .001), and when CNB Ki67s were obtained by manual counting, the CR for Ki67 was 76.2\% (kappa, 0.534; p <.001) (Table 4).

\section{Concordance of molecular subtypes}

Molecular subtypes demonstrated good agreement (kappa, 0.672 in four subtypes and kappa, 0.696 in five subtypes). CRs of surgical specimens with $\mathrm{CNB}$ s for luminal A, luminal B, HER2, and triple negative were $89.0 \%, 70.0 \%, 82.9 \%$, and $77.2 \%$, respectively (kappa, 0.672 ; good agreement). The CRs of luminal A and HER2 showed significant agreement; however, luminal B showed the lowest $\mathrm{CR}$ among the molecular subtypes. When luminal B was subdivided into luminal B-HER2-negative and -HER2-positive, the CRs for these receptors between $\mathrm{CNB}$ and surgical specimens were $61.7 \%$ and $95.1 \%$, respectively. Therefore, the especially high discrepancy seen for luminal B-HER2-negative occurred because molecular subtypes changed from luminal A due to low $\mathrm{Ki} 67$ in $\mathrm{CNB}$ (75 cases) to luminal B-HER2-negative after high Ki67 was found in surgical specimens (Tables 5, 6).

\section{DISCUSSION}

Neoadjuvant therapy in breast cancer can reduce tumor burden, which facilitates breast conserving surgery, preserves the axilla, and identifies response to systemic therapy before surgery [5-7]. Typically, treatment policies for neoadjuvant systemic therapy are decided according to the molecular subtype determined by ER, PR, HER2, and Ki67 status of CNB. These are also used to predict prognoses of breast cancer patients. In this study, the CRs of ER, PR, HER2, and Ki67 status in CNB specimens prior to surgery and those from surgical specimens were analyzed to confirm the accuracy of molecular classifications performed by CNB. ER and PR status showed high CRs of 96.5\% (kappa, 0.883) and 93.0\% (kappa, 0.824), respectively (Table 3). However, a greater distribution and bigger change of

Table 4. Comparing Ki67 cutoff values between CNB and surgical specimens by digital image analysis and manual scoring assessment

\begin{tabular}{|c|c|c|c|c|c|c|c|c|c|c|c|c|}
\hline \multirow{3}{*}{ Ki67 } & \multicolumn{6}{|c|}{ Digital image analysis } & \multicolumn{6}{|c|}{ Manual counting method } \\
\hline & \multicolumn{3}{|c|}{ CNB } & \multirow{2}{*}{$\begin{array}{c}\text { Concordance } \\
\text { rate (\%) }\end{array}$} & \multirow{2}{*}{ Kappa $^{a}$} & \multirow{2}{*}{$p$-value } & \multicolumn{3}{|c|}{ CNB } & \multirow{2}{*}{$\begin{array}{c}\text { Concordance } \\
\text { rate (\%) }\end{array}$} & \multirow{2}{*}{ Kappa $^{a}$} & \multirow{2}{*}{$p$-value } \\
\hline & $\leq 20 \%$ & $>20 \%$ & Total & & & & $\leq 20 \%$ & $>20 \%$ & Total & & & \\
\hline$\leq 20 \%$ & 88 & 28 & 116 & 82.3 & 0.639 & $<.001$ & 137 & 73 & 210 & 76.2 & 0.534 & $<.001$ \\
\hline$>20 \%$ & 18 & 126 & 144 & & & & 15 & 144 & 159 & & & \\
\hline Total & 106 & 154 & 260 & & & & 152 & 217 & 369 & & & \\
\hline
\end{tabular}

CNB, core needle biopsy.

aKappa: $<0.20$, poor agreement; $0.21-0.40$, fair agreement; $0.41-0.60$, moderate agreement; $0.61-0.80$, good agreement; $0.81-1.00$, very good agreement.

Table 5. Concordance between CNB and surgical specimens for molecular subtypes

\begin{tabular}{|c|c|c|c|c|c|c|c|c|}
\hline Surgical specimen & CNB & Luminal A & Luminal B & Triple negative & HER2+ & Total & Concordance rate (\%) & Kappa \\
\hline Luminal A & & 218 & 26 & 1 & 0 & 245 & 89.0 & 0.672 \\
\hline Luminal B & & 75 & 189 & 4 & 2 & 270 & 70.0 & \\
\hline HER2+ & & 0 & 6 & 0 & 29 & 35 & 82.9 & \\
\hline Triple negative & & 2 & 16 & 61 & 0 & 79 & 77.2 & \\
\hline Total & & 295 & 237 & 66 & 31 & 629 & 79.0 & \\
\hline
\end{tabular}

CNB, core needle biopsy; HER2, human epidermal growth factor receptor 2.

Table 6. Concordance between CNB and surgical specimens for molecular subtypes including the subdivision of luminal B

\begin{tabular}{|c|c|c|c|c|c|c|c|c|c|}
\hline Surgical specimen & CNB & Luminal A & $\begin{array}{c}\text { Luminal B } \\
\text { (HER2-) }\end{array}$ & $\begin{array}{l}\text { Luminal B } \\
\text { (HER2+) }\end{array}$ & Triple negative & HER2+ & Total & Concordance rate (\%) & Kappa \\
\hline Luminal A & & 218 & 26 & 0 & 1 & 0 & 245 & 89.0 & 0.696 \\
\hline Luminal B (HER2-) & & 75 & 129 & 1 & 4 & 0 & 209 & 61.7 & \\
\hline Luminal B (HER2+) & & 0 & 1 & 58 & 0 & 2 & 61 & 95.1 & \\
\hline HER2+ & & 0 & 0 & 6 & 0 & 29 & 35 & 82.9 & \\
\hline Triple negative & & 2 & 16 & 0 & 61 & 0 & 79 & 77.2 & \\
\hline Total & & 295 & 172 & 65 & 66 & 31 & 629 & 78.7 & \\
\hline
\end{tabular}

CNB, core needle biopsy; HER2, human epidermal growth factor receptor 2. 
Allred scores from CNB to surgical specimen were noted for PR than ER (Figs. 1, 2). These findings are consistent with previous reports $[16,17]$ and suggest that the reason behind these findings is heterogeneity of PR expression in tumor cells.

Identifying HER2-positive breast cancer patients by $\mathrm{CNB}$ is important, as neoadjuvant HER2-targeted therapy is an effective option for these patients. In this study, the CR for HER2 status, as determined by HER2 IHC or reflex HER2 SISH, was as high as $99.7 \%$ (kappa, 0.988), which was higher than most previous reports (ranging from 61\%-97.3\%) [10,18-29]. The reason for this high CR could partially be due to performing reflex HER2 SISH for all HER2 IHC equivocal cases to determine final HER2 status strictly following ASCO/CAP guidelines. Sufficient CNB specimens were obtained when radiological microcalcifications were noted. In our hospital, at least four core passes in CNB are usually obtained by ultrasound guidance with a 14-gauge needle. However, when radiological calcifications were identified, additional core passes (at least seven) were performed. Sometimes when scattered calcification was noted, ultrasound-guided mammotome biopsy was performed with an 11-gauge needle, and $>12$ core passes were obtained. It has been reported that radiologically recognized calcifications in breast cancer are associated with HER2 molecular subtype and pCR after neoadjuvant chemotherapy [30,31]. Another reason for collection of multiple cores in CNB is to overcome tumor heterogeneity. For accurate histologic diagnoses of breast cancer, at least four cores should be obtained using a 14-gauge needle [32]. It has been reported that the accuracy of histologic diagnoses (including tumor grade) plateaus at $74 \%$ when four passes are performed, while accuracy is only 32\% with one pass [33]. Greer et al. [34] reported that the concordance for ER and PR between $\mathrm{CNB}$ and surgical specimen improved with increasing numbers of core passes. However, they also found the concordance of HER2 to be limited when tumor heterogeneity was present [34].

Although ER, PR, and HER2 status showed high CRs between CNB and surgical specimen, Ki67 revealed only moderate agreement. There were 75 cases classified as luminal A from CNB that were moved to luminal B after evaluating surgical specimens, as Ki67 was higher in the surgical specimen than in CNB. Additionally, a higher median value for Ki67 was identified in the surgical specimens (Tables 3, 5, 6). The tendency for a greater Ki67 labeling index in surgical specimens than in CNB has been reported in several studies. The authors explained that it was due to tumor heterogeneity $[15,27]$. Another study reported that the $\mathrm{CR}$ for Ki67 between CNB and surgical specimen improved slightly with increased number of core passes to account for tumor heterogeneity; however, after more than six core passes, a plateau was reached [34]. In the current study, the Ki67 labeling index was obtained by digital image analysis or manual scoring (Table 4). Digital image analysis of Ki67 in CNB showed better concordance with surgical specimen $\mathrm{Ki} 67$ (CR, 82.3\% vs. 76.2 ; kappa, 0.639 vs. 0.534 , respectively).

Finally, $21.0 \%$ of cases switched molecular subtype between $\mathrm{CNB}$ and surgical specimen, mostly because Ki67 changed from low to high; for example, 75 luminal $A$ cases as assessed by $\mathrm{CNB}$ were moved to luminal $\mathrm{B}$ after evaluating surgical specimens (Table 5). There were also a few cases where hormone status changed from positive to negative, 16 cases of luminal B changed to triple negative, and six cases changed to HER2. The reason for these discrepancies could be fixation conditions such as delayed, under-, or over-fixation with formalin; crush artifacts by needle sampling; or tumor heterogeneity.

In conclusion, $\mathrm{CNB}$ with adequate core passes can be reliably used to access molecular subtypes for systemic treatment in invasive breast cancer. Digital image analysis of Ki67 should be used to achieve better, more accurate molecular classifications from CNBs.

\section{ORCID}

Ye Sul Jeong: https://orcid.org/0000-0003-2952-1662

Jun Kang: https://orcid.org/0000-0002-7967-0917

Jieun Lee: https://orcid.org/0000-0002-2656-0650

Tae-Kyung Yoo: https://orcid.org/0000-0002-5790-353X

Sung Hun Kim: https://orcid.org/0000-0003-4478-9720

Ahwon Lee: https://orcid.org/0000-0002-2523-9531

\section{Author Contributions}

Conceptualization: AL.

Data curation: YSJ, TKY.

Formal analysis: YSJ, SHK, AL.

Methodology: YSJ, SHK, JK, AL.

Project administration: YSJ.

Resources: AL.

Supervision: AL.

Validation: YSJ, JL, AL.

Visualization: YSJ.

Writing—original draft: YSJ.

Writing —review \& editing: YSJ, SHK, JK, AL.

\section{Conflicts of Interest}

The authors declare that they have no potential conflicts of 
interest.

\section{Funding}

No funding to declare.

\section{REFERENCES}

1. Cheang MC, Chia SK, Voduc D, et al. Ki67 index, HER2 status, and prognosis of patients with luminal B breast cancer. J Natl Cancer Inst 2009; 101: 736-50.

2. Goldhirsch A, Winer EP, Coates AS, et al. Personalizing the treatment of women with early breast cancer: highlights of the St Gallen International Expert Consensus on the Primary Therapy of Early Breast Cancer 2013. Ann Oncol 2013; 24: 2206-23.

3. Goldhirsch A, Wood WC, Coates AS, et al. Strategies for subtypes: dealing with the diversity of breast cancer: highlights of the St. Gallen International Expert Consensus on the Primary Therapy of Early Breast Cancer 2011. Ann Oncol 2011; 22: 1736-47.

4. Neubauer H, Gall C, Vogel U, et al. Changes in tumour biological markers during primary systemic chemotherapy (PST). Anticancer Res 2008; 28: 1797-804.

5. Untch M, Konecny GE, Paepke S, von Minckwitz G. Current and future role of neoadjuvant therapy for breast cancer. Breast 2014; 23: 526-37.

6. Teshome M, Hunt KK. Neoadjuvant therapy in the treatment of breast cancer. Surg Oncol Clin N Am 2014; 23: 505-23.

7. Loibl S, Denkert C, von Minckwitz G. Neoadjuvant treatment of breast cancer: clinical and research perspective. Breast 2015; 24 Suppl 2: S73-7.

8. Ge WK, Yang B, Zuo WS, et al. Evaluation of hormone receptor, human epidermal growth factor receptor-2 and Ki-67 with core needle biopsy and neoadjuvant chemotherapy effects in breast cancer patients. Thorac Cancer 2015; 6: 64-9.

9. Piper GL, Patel NA, Patel JA, Malay MB, Julian TB. Neoadjuvant chemotherapy for locally advanced breast cancer results in alterations in preoperative tumor marker status. Am Surg 2004; 70: 1103-6.

10. Meattini I, Bicchierai G, Saieva C, et al. Impact of molecular subtypes classification concordance between preoperative core needle biopsy and surgical specimen on early breast cancer management: single-institution experience and review of published literature. Eur J Surg Oncol 2017; 43: 642-8.

11. Allred DC, Harvey JM, Berardo M, Clark GM. Prognostic and predictive factors in breast cancer by immunohistochemical analysis. Mod Pathol 1998; 11: 155-68.

12. Wolff AC, Hammond ME, Allison KH, et al. Human epidermal growth factor receptor 2 testing in breast cancer: American Society of Clinical Oncology/College of American Pathologists Clinical Practice Guideline Focused Update. J Clin Oncol 2018; 36: 2105-22.

13. Wolff AC, Hammond ME, Hicks DG, et al. Recommendations for human epidermal growth factor receptor 2 testing in breast cancer: American Society of Clinical Oncology/College of American Pathologists clinical practice guideline update. J Clin Oncol 2013; 31: 3997-4013.

14. Cho U, Kim HE, Oh WJ, Yeo MK, Song BJ, Lee A. The long-term prognostic performance of Ki-67 in primary operable breast cancer and evaluation of its optimal cutoff value. Appl Immunohistochem Mol Morphol 2016; 24: 159-66.

15. Dowsett M, Nielsen TO, A'Hern R, et al. Assessment of Ki67 in breast cancer: recommendations from the International Ki67 in Breast Cancer working group. J Natl Cancer Inst 2011; 103: 1656-64.

16. Zidan A, Christie Brown JS, Peston D, Shousha S. Oestrogen and progesterone receptor assessment in core biopsy specimens of breast carcinoma. J Clin Pathol 1997; 50: 27-9.

17. Rakha EA, Ellis IO. An overview of assessment of prognostic and predictive factors in breast cancer needle core biopsy specimens. J Clin Pathol 2007; 60: 1300-6.

18. Park SY, Kim KS, Lee TG, et al. The accuracy of preoperative core biopsy in determining histologic grade, hormone receptors, and human epidermal growth factor receptor 2 status in invasive breast cancer. Am J Surg 2009; 197: 266-9.

19. Ricci MD, Calvano Filho CM, Oliveira Filho HR, Filassi JR, Pinotti JA, Baracat EC. Analysis of the concordance rates between core needle biopsy and surgical excision in patients with breast cancer. Rev Assoc Med Bras (1992) 2012; 58: 532-6.

20. Ozdemir A, Voyvoda NK, Gultekin S, Tuncbilek I, Dursun A, Yamac D. Can core biopsy be used instead of surgical biopsy in the diagnosis and prognostic factor analysis of breast carcinoma? Clin Breast Cancer 2007; 7: 791-5.

21. Ough M, Velasco J, Hieken TJ. A comparative analysis of core needle biopsy and final excision for breast cancer: histology and marker expression. Am J Surg 2011; 201: 692-4.

22. Chen J, Wang Z, Lv Q, et al. Comparison of core needle biopsy and excision specimens for the accurate evaluation of breast cancer molecular markers: a report of 1003 cases. Pathol Oncol Res 2017; 23: 769-75.

23. You K, Park S, Ryu JM, et al. Comparison of core needle biopsy and surgical specimens in determining intrinsic biological subtypes of breast cancer with immunohistochemistry. J Breast Cancer 2017; 20: 297-303.

24. Ensani F, Omranipour R, Jahanzad I, Jafari A, Nafarzadeh S, Aminishakib P. The core needle and surgical biopsy concordance to detect estrogen, progesterone, and Her-2 receptors in breast cancer: a com- 
parative study. Iran J Pathol 2017; 12: 202-8.

25. Liu M, Tang SX, Tsang JY, et al. Core needle biopsy as an alternative to whole section in IHC4 score assessment for breast cancer prognostication. J Clin Pathol 2018; 71: 1084-9.

26. Lorgis V, Algros MP, Villanueva C, et al. Discordance in early breast cancer for tumour grade, estrogen receptor, progesteron receptors and human epidermal receptor-2 status between core needle biopsy and surgical excisional primary tumour. Breast 2011; 20: 284-7.

27. Chen X, Sun L, Mao Y, et al. Preoperative core needle biopsy is accurate in determining molecular subtypes in invasive breast cancer. BMC Cancer 2013; 13: 390.

28. Usami S, Moriya T, Amari M, et al. Reliability of prognostic factors in breast carcinoma determined by core needle biopsy. Jpn J Clin Oncol 2007; 37: 250-5.

29. Robertson S, Ronnlund C, de Boniface J, Hartman J. Re-testing of predictive biomarkers on surgical breast cancer specimens is clinically relevant. Breast Cancer Res Treat 2019; 174: 795-805.
30. Mazari FA, Sharma N, Dodwell D, Horgan K. Human epidermal growth factor 2-positive breast cancer with mammographic microcalcification: relationship to pathologic complete response after neoadjuvant chemotherapy. Radiology 2018; 288: 366-74.

31. Nie Z, Wang J, Ji XC. Microcalcification-associated breast cancer: HER2-enriched molecular subtype is associated with mammographic features. Br J Radiol 2018 Jun 21 [Epub]. https://doi.org/10.1259/ bjr.20170942.

32. Fishman JE, Milikowski C, Ramsinghani R, Velasquez MV, Aviram G. US-guided core-needle biopsy of the breast: how many specimens are necessary? Radiology 2003; 226: 779-82.

33. Mcllhenny C, Doughty JC, George WD, Mallon EA. Optimum number of core biopsies for accurate assessment of histological grade in breast cancer. Br J Surg 2002; 89: 84-5.

34. Greer LT, Rosman M, Mylander WC, et al. Does breast tumor heterogeneity necessitate further immunohistochemical staining on surgical specimens? J Am Coll Surg 2013; 216: 239-51. 